\title{
A FOURIER APPROACH TO THE PROFILE DECOMPOSITION IN ORLICZ SPACES
}

\author{
Hajer Bahouri and Galina Perelman
}

\begin{abstract}
This paper is devoted to the characterization of the lack of compactness of the Sobolev embedding of $H^{N}\left(\mathbb{R}^{2 N}\right)$ into the Orlicz space using Fourier analysis. The approach adopted in this paper is strikingly different from the one used in 2D in $[4,6,7]$, which consists in tracking the large values of the sequences considered. The analysis we employ in this work is inspired by the strategy of P. Gérard in [13] and is based on the notion introduced in [8] of being log-oscillating with respect to a scale.
\end{abstract}

\section{Introduction and statement of the results}

1.1. Setting of the problem and main result. We are interested in the nonhomogeneous Sobolev space $H^{N}\left(\mathbb{R}^{2 N}\right)$ which consists of functions $u$ in $L^{2}\left(\mathbb{R}^{2 N}\right)$ such that

$$
\|u\|_{H^{N}\left(\mathbb{R}^{2 N}\right)}^{2}:=\int_{\mathbb{R}^{2 N}}\left(1+|\xi|^{2}\right)^{N}|\widehat{u}(\xi)|^{2} d \xi<\infty
$$

where $\widehat{u}$ denotes the Fourier transform of $u$ :

$$
\widehat{u}(\xi):=\int_{\mathbb{R}^{2 N}} \mathrm{e}^{-i x \cdot \xi} u(x) d x,
$$

with $x \cdot \xi$ the inner product on $\mathbb{R}^{2 N}$. It is well known that $H^{N}\left(\mathbb{R}^{2 N}\right)$ is continuously embedded in all Lebesgue spaces $L^{p}\left(\mathbb{R}^{2 N}\right)$ for $2 \leq p<\infty$, but not in $L^{\infty}\left(\mathbb{R}^{2 N}\right)$. On the other hand, it is also known that $H^{N}\left(\mathbb{R}^{2 N}\right)$ embeds in $\mathcal{L}\left(\mathbb{R}^{2 N}\right)$, where $\mathcal{L}\left(\mathbb{R}^{2 N}\right)$ denotes the Orlicz space associated to the function $\phi(s)=\mathrm{e}^{s^{2}}-1$. Recall that the Orlicz spaces are defined as follows:

Definition 1.1. Let $\phi: \mathbb{R}^{+} \rightarrow \mathbb{R}^{+}$be a convex increasing function such that

$$
\phi(0)=0=\lim _{s \rightarrow 0^{+}} \phi(s), \quad \lim _{s \rightarrow \infty} \phi(s)=\infty .
$$

We say that a measurable function $u: \mathbb{R}^{d} \rightarrow \mathbb{C}$ belongs to $L^{\phi}$ if there exists $\lambda>0$ such that

$$
\int_{\mathbb{R}^{d}} \phi\left(\frac{|u(x)|}{\lambda}\right) d x<\infty
$$

We denote then

$$
\|u\|_{L^{\phi}}=\inf \left\{\lambda>0, \quad \int_{\mathbb{R}^{d}} \phi\left(\frac{|u(x)|}{\lambda}\right) d x \leq 1\right\} .
$$

Received by the editors July 1, 2013.

Key words and phrases. Orlicz; lack of compactness; profile decomposition. 
The Sobolev embedding

$$
H^{N}\left(\mathbb{R}^{2 N}\right) \hookrightarrow \mathcal{L}\left(\mathbb{R}^{2 N}\right)
$$

stems immediately from the following sharp Moser-Trudinger type inequalities (see $[1,2,23,24]$ for further details):

\section{Proposition 1.2.}

$$
\sup _{\|u\|_{H^{N}\left(\mathbb{R}^{2 N}\right)} \leq 1} \int_{\mathbb{R}^{2 N}}\left(\mathrm{e}^{\beta_{N}|u(x)|^{2}}-1\right) d x<\infty,
$$

where $\beta_{N}=\frac{2 N \pi^{2 N} 2^{2 N}}{\omega_{2 N-1}}$, with $\omega_{2 N-1}=\frac{2 \pi^{N}}{(N-1) !}$ the measure of the sphere $\mathbb{S}^{2 N-1}$.

The aim of this paper is to describe the lack of compactness of the Sobolev embedding of $H^{N}\left(\mathbb{R}^{2 N}\right)$ into $\mathcal{L}\left(\mathbb{R}^{2 N}\right)$. In $[4,6,7]$, the authors studied this question in the two-dimensional (2D) particular case and characterized the lack of compactness by means of an asymptotic decomposition in terms of generalizations of the following example by Moser ( [20-22]):

$$
f_{\alpha_{n}}(x)=\left\{\begin{array}{lll}
\sqrt{\frac{\alpha_{n}}{2 \pi}} & \text { if } & |x| \leq \mathrm{e}^{-\alpha_{n}}, \\
-\frac{\log |x|}{\sqrt{2 \alpha_{n} \pi}} & \text { if } & \mathrm{e}^{-\alpha_{n}} \leq|x| \leq 1, \\
0 & \text { if } \quad & |x| \geq 1,
\end{array}\right.
$$

where $\left(\alpha_{n}\right)$ is a sequence of positive real numbers going to infinity. In order to state in a clear way the corresponding result, let us introduce some notations as in [7].

Definition 1.3. We shall designate by a scale any sequence $\underline{\alpha}:=\left(\alpha_{n}\right)$ of positive real numbers going to infinity, by a core any sequence $\underline{x}:=\left(x_{n}\right)$ of points in $\mathbb{R}^{2 N}$ and by a profile any function $\varphi$ belonging to $L^{2}\left(\mathbb{R}_{+}\right)$. Given two scales $\underline{\alpha}, \underline{\tilde{\alpha}}$, two cores $\underline{x}, \underline{\tilde{x}}$ and two profiles $\varphi, \widetilde{\varphi}$, we shall say that the triplets $(\underline{\alpha}, \underline{x}, \varphi)$ and $(\underline{\tilde{\alpha}}, \underline{\tilde{x}}, \tilde{\varphi})$, are orthogonal if

$$
\text { either } \quad\left|\log \left(\tilde{\alpha}_{n} / \alpha_{n}\right)\right| \stackrel{n \rightarrow \infty}{\longrightarrow} \infty \text {, }
$$

or $\tilde{\alpha}_{n}=\alpha_{n}$ and

$$
-\frac{\log \left|x_{n}-\tilde{x}_{n}\right|}{\alpha_{n}} \stackrel{n \rightarrow \infty}{\longrightarrow} a \in[-\infty,+\infty[,
$$

with in the case when $a \in] 0,+\infty\left[\psi\right.$ or $\tilde{\psi}$ null for $s<a$, where $\psi(s):=\int_{0}^{s} \varphi(t) d t$ and $\tilde{\psi}(s):=\int_{0}^{s} \tilde{\varphi}(t) d t$.

In [7], the following result is proved.

Theorem 1.4. Let $\left(u_{n}\right)$ be a bounded sequence in $H^{1}\left(\mathbb{R}^{2}\right)$ such that

$$
\begin{aligned}
& u_{n} \rightarrow 0 \quad \text { in } H^{1}\left(\mathbb{R}^{2}\right), \quad n \rightarrow \infty, \\
& \limsup _{n \rightarrow \infty}\left\|u_{n}\right\|_{\mathcal{L}}=A_{0}>0 \quad \text { and }
\end{aligned}
$$




$$
\lim _{R \rightarrow \infty} \limsup _{n \rightarrow \infty}\left\|u_{n}\right\|_{\mathcal{L}(|x|>R)}=0 .
$$

Then, up to a subsequence extraction, there exists a sequence of scales $\left(\underline{\alpha}^{(j)}\right)$, a sequence of cores $\left(\underline{x}^{(j)}\right)$ and a sequence of profiles $\left(\varphi^{(j)}\right)$ such that the triplets $\left(\underline{\alpha}^{(j)}, \underline{x}^{(j)}\right.$, $\left.\varphi^{(j)}\right)$ are pairwise orthogonal in the sense of Definition 1.3 and, we have for all $\ell \geq 1$, $(1.10)$

$$
u_{n}(x)=\sum_{j=1}^{\ell} \sqrt{\frac{\alpha_{n}^{(j)}}{2 \pi}} \psi^{(j)}\left(\frac{-\log \left|x-x_{n}^{(j)}\right|}{\alpha_{n}^{(j)}}\right)+r_{n}^{(\ell)}(x), \quad \limsup _{n \rightarrow \infty}\left\|r_{n}^{(\ell)}\right\|_{\mathcal{L}} \stackrel{\ell \rightarrow \infty}{\longrightarrow} 0,
$$

where $\psi^{(j)}(s):=\int_{0}^{s} \varphi^{(j)}(t) d t$. Moreover for all $\ell \geq 1$, we have the following orthogonality equality

$$
\left\|\nabla u_{n}\right\|_{L^{2}\left(\mathbb{R}^{2}\right)}^{2}=\sum_{j=1}^{\ell}\left\|\varphi^{(j)}\right\|_{L^{2}\left(\mathbb{R}_{+}\right)}^{2}+\left\|\nabla r_{n}^{(\ell)}\right\|_{L^{2}\left(\mathbb{R}^{2}\right)}^{2}+\circ(1), \quad n \rightarrow \infty .
$$

\section{Remarks 1.5.}

- The example by Moser reads as

$$
f_{\alpha_{n}}(x)=\sqrt{\frac{\alpha_{n}}{2 \pi}} \mathbf{L}\left(\frac{-\log |x|}{\alpha_{n}}\right),
$$

where

$$
\mathbf{L}(s)= \begin{cases}0 & \text { if } \quad s \leq 0 \\ s & \text { if } \quad 0 \leq s \leq 1 \\ 1 & \text { if } \quad s \geq 1\end{cases}
$$

- It was shown in $[8]$ that the sequence $\left(f_{\alpha_{n}}\right)$ can be written under the form:

$$
f_{\alpha_{n}}(x)=\widetilde{f_{\alpha_{n}}}(x)+r_{n}(x),
$$

with $\left\|\nabla r_{n}\right\|_{L^{2}} \stackrel{n \rightarrow \infty}{\longrightarrow} 0$ and

$$
\widetilde{f_{\alpha_{n}}}(x)=\frac{1}{(2 \pi)^{2}} \sqrt{\frac{2 \pi}{\alpha_{n}}} \int_{\mathbb{R}^{2}} \mathrm{e}^{i x \cdot \xi} \frac{1}{|\xi|^{2}} \varphi\left(\frac{\log |\xi|}{\alpha_{n}}\right) d \xi,
$$

where $\varphi(\eta)=\mathbf{1}_{[0,1]}(\eta)$.

Let us point out that the strategy adopted in [7] to build the asymptotic decomposition in terms of generalizations of the example by Moser concentrated around cores is based on capacity arguments and uses in a crucial way the fact that the Schwarz symmetrization minimizes the energy (for more details, we refer the reader to $[17,19]$ and references therein). As the Schwarz symmetrization process does not allow to control the $H^{N}$-norm when $N>1$, the method developed in [7] does not apply to higher dimensions. 
The strategy we adopt here to describe the lack of compactness of the Sobolev embedding (1.3) in the 2ND general case is rather inspired by the approach of P. Gérard in [13] which is based on Fourier analysis. However, unlike the framework studied by P. Gérard, the elements which are responsible of the lack of compactness of (1.3) are spread in frequency. We are therefore led to revisit the method of P. Gérard to address that issue.

Note that the description of the lack of compactness in other critical Sobolev embeddings was achieved in $[5,10,13,14]$ and has been at the origin of several developments such as regularity results for Navier-Stokes systems in $[9,12]$, qualitative study of nonlinear evolution equations in $[3,16,18,26]$ or estimate of the life span of focusing semi-linear dispersive evolution equations in [15]. Further applications of the characterization of the lack of compactness in critical Sobolev embeddings are available in the elliptic framework. Among others, one can mention [11,20,21, 25].

The main purpose of this paper is to establish the following theorem:

Theorem 1.6. Let $\left(u_{n}\right)$ be a bounded sequence in $H^{N}\left(\mathbb{R}^{2 N}\right)$ such that

$$
\begin{gathered}
u_{n} \rightarrow 0 \quad \text { in } H^{N}\left(\mathbb{R}^{2 N}\right), \quad n \rightarrow \infty, \\
\limsup _{n \rightarrow \infty}\left\|u_{n}\right\|_{\mathcal{L}}=A_{0}>0 \quad \text { and } \\
\lim _{R \rightarrow \infty} \limsup _{n \rightarrow \infty} \int_{|x| \geq R}\left|u_{n}\right|^{2} d x=0 .
\end{gathered}
$$

Then, there exists a sequence of scales $\left(\underline{\alpha}^{(j)}\right)$, a sequence of cores $\left(\underline{x}^{(j)}\right)$ and a sequence of profiles $\left(\varphi^{(j)}\right)$ such that the triplets $\left(\underline{\alpha}^{(j)}, \underline{x}^{(j)}, \varphi^{(j)}\right)$ are pairwise orthogonal in the sense of Definition 1.3 and such that, up to a subsequence extraction, we have for all $\ell \geq 1$,

$$
u_{n}(x)=\sum_{j=1}^{\ell} \frac{C_{N}}{\sqrt{\alpha_{n}^{(j)}}} \int_{|\xi| \geq 1} \frac{\mathrm{e}^{i\left(x-x_{n}^{(j)}\right) \cdot \xi}}{|\xi|^{2 N}} \varphi^{(j)}\left(\frac{\log |\xi|}{\alpha_{n}^{(j)}}\right) d \xi+r_{n}^{(\ell)}(x),
$$

with $C_{N}=\frac{1}{(2 \pi)^{N} \sqrt{\omega_{2 N-1}}}$ and $\limsup _{n \rightarrow \infty}\left\|r_{n}^{(\ell)}\right\|_{\mathcal{L}} \stackrel{\ell \rightarrow \infty}{\longrightarrow} 0$.

Moreover for all $\ell \geq 1$, we have the following stability estimates

$$
\left\|u_{n}\right\|_{\dot{H}^{N}\left(\mathbb{R}^{2 N}\right)}^{2}=\sum_{j=1}^{\ell}\left\|\varphi^{(j)}\right\|_{L^{2}\left(\mathbb{R}_{+}\right)}^{2}+\left\|r_{n}^{(\ell)}\right\|_{\dot{H}^{N}\left(\mathbb{R}^{2 N}\right)}^{2}+\circ(1), \quad n \rightarrow \infty .
$$

The following proposition the proof of which is postponed to Appendix A.2 allows us to relate in the $2 \mathrm{D}$ case Theorem 1.6 to Theorem 1.4.

Proposition 1.7. Let $\left(\alpha_{n}\right)_{n \geq 0}$ be a scale in the sense of Definition 1.3 and $\varphi$ in $L^{2}\left(\mathbb{R}_{+}\right)$. Set with the notation of Theorem 1.6

$$
g_{n}(x):=\frac{C_{N}}{\sqrt{\alpha_{n}}} \int_{|\xi| \geq 1} \frac{\mathrm{e}^{i x \cdot \xi}}{|\xi|^{2 N}} \varphi\left(\frac{\log |\xi|}{\alpha_{n}}\right) d \xi
$$


Then

$$
g_{n}(x)=\widetilde{C}_{N} \sqrt{\alpha_{n}} \psi\left(\frac{-\log |x|}{\alpha_{n}}\right)+t_{n}(x),
$$

with $\psi(y)=\int_{0}^{y} \varphi(t) d t, \widetilde{C}_{N}=\frac{\sqrt{\omega_{2 N-1}}}{(2 \pi)^{N}}$ and $\left\|t_{n}\right\|_{\mathcal{L}} \stackrel{n \rightarrow \infty}{\longrightarrow} 0$.

By this proposition, one can write the elementary concentrations

$$
f_{n}^{(j)}(x):=\frac{C_{N}}{\sqrt{\alpha_{n}^{(j)}}} \int_{|\xi| \geq 1} \frac{\mathrm{e}^{i\left(x-x_{n}^{(j)}\right) \cdot \xi}}{|\xi|^{2 N}} \varphi^{(j)}\left(\frac{\log |\xi|}{\alpha_{n}^{(j)}}\right) d \xi,
$$

under the form

$$
f_{n}^{(j)}(x)=\widetilde{C}_{N} \sqrt{\alpha_{n}^{(j)}} \psi^{(j)}\left(\frac{-\log \left|x-x_{n}^{(j)}\right|}{\alpha_{n}^{(j)}}\right)+t_{n}^{(j)}(x),
$$

where $\widetilde{C}_{N}=\frac{\sqrt{\omega_{2 N-1}}}{(2 \pi)^{N}}$ and $\left\|t_{n}^{(j)}\right\|_{\mathcal{L}} \stackrel{n \rightarrow \infty}{\longrightarrow} 0$. This allows us to view Theorem 1.6 as a generalization of Theorem 1.4.

\section{Remarks 1.8.}

- Arguing exactly as in the proof of Proposition 1.15 in [4] and making use of (1.4) and (1.20), we can prove that the elementary concentrations $f_{n}^{(j)}$ satisfy

$$
\lim _{n \rightarrow \infty}\left\|f_{n}^{(j)}\right\|_{\mathcal{L}}=\frac{1}{\sqrt{\beta_{N}}} \max _{s>0} \frac{\left|\psi^{(j)}(s)\right|}{\sqrt{s}},
$$

where $\psi^{(j)}(s):=\int_{0}^{s} \varphi^{(j)}(t) d t$

- Note that the elementary concentrations $f_{n}^{(j)}$ defined above by (1.19) belong to $H^{N}\left(\mathbb{R}^{2 N}\right)$ while a priori the generalizations of the example by Moser:

$\widetilde{C}_{N} \sqrt{\alpha_{n}^{(j)}} \psi^{(j)}\left(\frac{-\log \left|x-x_{n}^{(j)}\right|}{\alpha_{n}^{(j)}}\right)$ only belong to $H^{1}\left(\mathbb{R}^{2 N}\right)$.

- The hypothesis of compactness at infinity (1.15) is crucial: it allows to avoid the loss of Orlicz norm at infinity; without this assumption, the result is not true.

- Note also that the lack of compactness of the Sobolev embedding (1.3) was studied in the radial framework for the $4 \mathrm{D}$ case in [10] by tracking the large values of the sequences studied.

1.2. Layout of the paper. The paper is organized as follows: in Section 2, we recall some useful results about profile decompositions. The proof of Theorem 1.6 is addressed in Section 3. In Appendix A, we establish Proposition 1.7 and highlight the connection between Orlicz space and some space involved in the proof of Theorem 1.6.

We mention that the letter $C$ will be used to denote an absolute constant which may vary from line to line. We also use $A \lesssim B$ to denote an estimate of the form 
$A \leq C B$ for some absolute constant $C$. For simplicity, we shall also still denote by $\left(u_{n}\right)$ any subsequence of $\left(u_{n}\right)$ and designate by $\circ(1)$ any sequence which tends to 0 as $n$ goes to infinity.

\section{Background material}

Let us start by introducing the notions of being log-oscillating with respect to a scale and of being log-unrelated to any scale, which are a natural adaptation to our setting of the vocabulary of P. Gérard in [13].

Definition 2.1. Let $v:=\left(v_{n}\right)_{n \geq 0}$ be a bounded sequence in $L^{2}\left(\mathbb{R}^{d}\right)$ and $\alpha:=\left(\alpha_{n}\right)_{n \geq 0}$ be a sequence of positive real numbers.

- The sequence $v$ is said $\alpha$ log-oscillating if

$$
\limsup _{n \rightarrow \infty}\left(\int_{|\xi| \leq \mathrm{e}^{\frac{\alpha_{n}}{R}}}\left|\widehat{v_{n}}(\xi)\right|^{2} d \xi+\int_{|\xi| \geq \mathrm{e}^{R \alpha_{n}}}\left|\widehat{v_{n}}(\xi)\right|^{2} d \xi\right) \stackrel{R \rightarrow \infty}{\longrightarrow} 0 .
$$

- The sequence $v$ is said $\log$-unrelated to the scale $\alpha$ if for any real numbers $b>a>0$

$$
\int_{\mathrm{e}^{a \alpha_{n}} \leq|\xi| \leq \mathrm{e}^{b \alpha_{n}}}\left|\widehat{v_{n}}(\xi)\right|^{2} d \xi \stackrel{n \rightarrow \infty}{\longrightarrow} 0
$$

\section{Remarks 2.2.}

- In what follows, it will be convenient for us to write in the $2 \mathrm{ND}$ case $^{1}$

$$
\widehat{v_{n}}(\xi)=\frac{1}{|\xi|^{N}} \varphi_{n}(\log |\xi|, \omega)
$$

Note that if $\left(v_{n}\right)_{n \in \mathbb{N}}$ is a bounded sequence in $L^{2}\left(\mathbb{R}^{2 N}\right)$ whose Fourier transform is supported in $\left\{\xi \in \mathbb{R}^{2 N} ;|\xi| \geq 1\right\}$, then $\left(\varphi_{n}\right)_{n \in \mathbb{N}}$ is a bounded sequence in the space $L^{2}\left(\mathbb{R}_{+} \times \mathbb{S}^{2 N-1}\right)$. In terms of $\left(\varphi_{n}\right)$, the property for $\left(v_{n}\right)$ to be $\alpha$ log-oscillating can be written as:

$$
\limsup _{n \rightarrow \infty}\left(\int_{\mathbb{S}^{2 N-1}} \int_{0}^{\frac{\alpha_{n}}{R}}\left|\varphi_{n}(t, \omega)\right|^{2} d t d \omega+\int_{\mathbb{S}^{2 N-1}} \int_{\alpha_{n} R}^{\infty}\left|\varphi_{n}(t, \omega)\right|^{2} d t d \omega\right) \stackrel{R \rightarrow \infty}{\longrightarrow} 0 .
$$

We will say that $\left(\varphi_{n}\right)_{n \in \mathbb{N}}$ is $\frac{1}{\alpha_{n}}$-concentrated.

Similarly, the property for $\left(v_{n}\right)$ being log-unrelated to the scale $\alpha$ means that for any real numbers $b>a>0$

$$
\int_{\mathbb{S}^{2 N-1}} \int_{a \alpha_{n}}^{b \alpha_{n}}\left|\varphi_{n}(t, \omega)\right|^{2} d t d \omega \stackrel{n \rightarrow \infty}{\longrightarrow} 0
$$

The sequence $\left(\varphi_{n}\right)_{n \in \mathbb{N}}$ will be said unrelated to the scale $\frac{1}{\alpha_{n}}$.

\footnotetext{
${ }^{1}$ Where obviously $\xi=|\xi| \cdot \omega$, with $\omega \in \mathbb{S}^{2 N-1}$.
} 
- According to (1.12), we can easily prove that the example by Moser $\nabla f_{\alpha_{n}}$ is $\alpha$ log-oscillating. Indeed for $R \geq 1$

$$
\int_{|\xi| \leq \mathrm{e}^{\frac{\alpha_{n}}{R}}}\left|\widehat{\nabla f_{\alpha_{n}}}(\xi)\right|^{2} d \xi=\int_{|\xi| \leq 1}\left|\widehat{\nabla f_{\alpha_{n}}}(\xi)\right|^{2} d \xi+\int_{1 \leq|\xi| \leq \mathrm{e}^{\frac{\alpha_{n}}{R}}}\left|\widehat{\nabla f_{\alpha_{n}}}(\xi)\right|^{2} d \xi .
$$

Firstly

$$
\int_{|\xi| \leq 1}\left|\widehat{\nabla f_{\alpha_{n}}}(\xi)\right|^{2} d \xi \leq\left\|f_{\alpha_{n}}\right\|_{L^{2}}^{2} \stackrel{n \rightarrow \infty}{\longrightarrow} 0,
$$

and secondly thanks to $(1.12)$

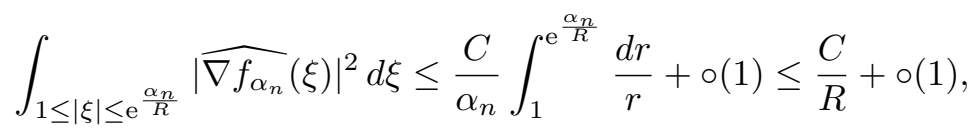

where $\circ(1)$ denotes a sequence which tends to 0 as $n$ goes to infinity. Therefore

$$
\limsup _{n \rightarrow \infty} \int_{|\xi| \leq \mathrm{e} \frac{\alpha_{n}}{R}}\left|\widehat{\nabla f_{\alpha_{n}}}(\xi)\right|^{2} d \xi \stackrel{R \rightarrow \infty}{\longrightarrow} 0 .
$$

Finally Identity (1.12) easily implies that

$$
\limsup _{n \rightarrow \infty} \int_{|\xi| \geq \mathrm{e}^{R \alpha_{n}}}\left|\widehat{\nabla f_{\alpha_{n}}}(\xi)\right|^{2} d \xi \stackrel{R \rightarrow \infty}{\longrightarrow} 0
$$

which ends the proof of the result.

In the proof of Theorem 1.6, we shall need the following result which is an immediate consequence of Theorem 2.9 in [13]. Note that in that framework a scale will designate a sequence $h:=\left(h_{n}\right)$ of positive real numbers and $\mathbf{1}$ the scale in which all the terms are equal to the number 1.

Theorem 2.3. Let $\left(\varphi_{n}\right)_{n \in \mathbb{N}}$ be a bounded sequence in $L^{2}\left(\mathbb{R}_{+} \times \mathbb{S}^{2 N-1}\right)$. Then, there exists a sequence of scales $\left(h^{(j)}\right)_{j \geq 1}$ and a sequence of bounded sequences $\left(g^{(j)}\right)_{j \geq 1}$ in the space $L^{2}\left(\mathbb{R}_{+} \times \mathbb{S}^{2 N-1}\right)$ such that:

- if $j \neq k,\left|\log \left(h_{n}^{(j)} / h_{n}^{(k)}\right)\right| \stackrel{n \rightarrow \infty}{\longrightarrow} \infty$;

- for all $j, g^{(j)}$ is $h^{(j)}$-concentrated;

- up to a subsequence extraction, we have for all $\ell \geq 1$

$$
\varphi_{n}(t, \omega)=\sum_{j=1}^{\ell} g_{n}^{(j)}(t, \omega)+r_{n}^{(\ell)}(t, \omega), \quad \text { with } \quad \limsup _{n \rightarrow \infty}\left\|r_{n}^{(\ell)}\right\|_{\mathcal{B}} \stackrel{\ell \rightarrow \infty}{\longrightarrow} 0,
$$

where

$$
\left\|r_{n}^{(\ell)}\right\|_{\mathcal{B}}:=\sup _{k \in \mathbb{Z}} \int_{\mathbb{S}^{2 N-1}} \int_{2^{k} \leq t \leq 2^{k+1}}\left|r_{n}^{(\ell)}(t, \omega)\right|^{2} d t d \omega
$$

Furthermore for any $\ell,\left(r_{n}^{(\ell)}\right)$ is unrelated to the scales $\left(h^{(j)}\right)$ for $j=1, \ldots, \ell$, and therefore

$$
\left\|\varphi_{n}\right\|_{L^{2}\left(\mathbb{R}_{+} \times \mathbb{S}^{2 N-1}\right)}^{2}=\sum_{j=1}^{\ell}\left\|g_{n}^{(j)}\right\|_{L^{2}\left(\mathbb{R}_{+} \times \mathbb{S}^{2 N-1}\right)}^{2}+\left\|r_{n}^{(\ell)}\right\|_{L^{2}\left(\mathbb{R}_{+} \times \mathbb{S}^{2 N-1}\right)}^{2}+\circ(1) .
$$




\section{Proof of Theorem 1.6}

3.1. Scheme of the proof. The proof relies on a diagonal extraction process and is done in three steps. In the first step, we extract the log-oscillating components of the sequence $\left(u_{n}\right)$ we investigate. For that purpose, we reduce the problem to the study of the bounded sequence $\left(\varphi_{n}\right)$ in $L^{2}\left(\mathbb{R}_{+} \times \mathbb{S}^{2 N-1}\right)$ expressed in terms of $\left(u_{n}\right)$ outside the low frequencies as follows:

$$
\widehat{u_{n}}(\xi)=\frac{1}{|\xi|^{2 N}} \varphi_{n}(\log |\xi|, \omega) .
$$

Then we extract the concentrated components of the sequence $\left(\varphi_{n}\right)$ applying Theorem 2.3. This allows us to complete this step expressing $\left(u_{n}\right)$ by means of $\left(\varphi_{n}\right)$.

The second step is dedicated to the extraction of the cores and the profiles. It consists firstly in applying with a slight modification the processes developed in [13], secondly in replacing the profiles obtained by this method in $L^{2}\left(\mathbb{R}_{+} \times \mathbb{S}^{2 N-1}\right)$ by their average on the sphere, and finally in translating the result in terms of $\left(u_{n}\right)$.

In the third step, we establish that the remainder term tends to zero in the Orlicz space as the number of functions in the sum and $n$ go to infinity. As we shall see in the sequel, the remainder term includes two parts. A first part which comes from the decomposition on log-oscillating components, and that we deal thanks to the connection between $\mathcal{B}$ space defined by (2.4) and Orlicz space. Regarding the second part, it comes from the error committed by replacing the profiles obtained in the second step by their average on the sphere. We treat it through some suitable estimates.

3.2. Extraction of log-oscillating components. In view of Assumption (1.15), the sequence $\left(u_{n}\right)$ converges towards 0 in $L^{2}\left(\mathbb{R}^{2 N}\right)$. Therefore, for any fixed $M$

$$
\int_{|\xi| \leq M}\left(1+|\xi|^{2}\right)^{N}\left|\widehat{u_{n}}(\xi)\right|^{2} d \xi \leq\left(1+M^{2}\right)^{N}\left\|u_{n}\right\|_{L^{2}\left(\mathbb{R}^{2 N}\right)}^{2} \stackrel{n \rightarrow \infty}{\longrightarrow} 0 .
$$

Thus writing ${ }^{2}$

$$
u_{n}=\chi(D) u_{n}+(1-\chi)(D) u_{n},
$$

where $\chi$ is a radial function in $\mathcal{D}\left(\mathbb{R}^{2 N}\right)$ equal to one in $B(0,1)$ and valued in $[0,1]$, we infer that

$$
u_{n}=\widetilde{u}_{n}+r_{n}^{1},
$$

where $\left(\widetilde{u}_{n}\right)$ is a bounded sequence in $H^{N}\left(\mathbb{R}^{2 N}\right)$ the Fourier transform of which is supported in $\left\{\xi \in \mathbb{R}^{2 N} ;|\xi| \geq 1\right\}$ and $\left\|r_{n}^{1}\right\|_{H^{N}\left(\mathbb{R}^{2 N}\right)} \stackrel{n \rightarrow \infty}{\longrightarrow} 0$.

Our starting point consists in observing that

$$
\widehat{\widetilde{u}_{n}}(\xi)=\frac{1}{|\xi|^{2 N}} \varphi_{n}(\log |\xi|, \omega),
$$

with $\varphi_{n}$ a bounded sequence of $L^{2}\left(\mathbb{R}_{+} \times \mathbb{S}^{2 N-1}\right)$. Indeed, we have

$$
\begin{aligned}
\| \mid \widehat{\left.D\right|^{N} \widetilde{u}_{n} \|_{L^{2}\left(\mathbb{R}^{2 N}\right)}^{2}} & =\int_{\mathbb{S}^{2 N-1}} d \omega \int_{1}^{\infty}\left|\varphi_{n}(\log \rho, \omega)\right|^{2} \frac{d \rho}{\rho} \\
& =\int_{\mathbb{S}^{2 N-1}} d \omega \int_{0}^{\infty}\left|\varphi_{n}(u, \omega)\right|^{2} d u,
\end{aligned}
$$

$2_{\text {we recall that }} \widehat{\chi(D) f}(\xi)=\chi(\xi) \widehat{f}(\xi)$. 
which ensures the result. Consequently, one can write

$$
u_{n}(x)=\frac{1}{(2 \pi)^{2 N}} \int_{|\xi| \geq 1} \frac{\mathrm{e}^{i x \cdot \xi}}{|\xi|^{2 N}} \varphi_{n}(\log |\xi|, \omega) d \xi+r_{n}^{1}(x),
$$

with $\left(\varphi_{n}\right)_{n \in \mathbb{N}}$ a bounded sequence in $L^{2}\left(\mathbb{R}_{+} \times \mathbb{S}^{2 N-1}\right)$. Taking advantage of the fact that $\left\|\widetilde{u}_{n}\right\|_{L^{2}\left(\mathbb{R}^{2 N}\right)} \stackrel{n \rightarrow \infty}{\longrightarrow} 0$, we infer with the vocabulary of Remarks 2.2 that the sequence $\left(\varphi_{n}\right)_{n \in \mathbb{N}}$ is unrelated to the scale $\mathbf{1}$ and to any scale $\left(h_{n}\right)_{n \in \mathbb{N}}$ tending to infinity. Indeed, for any real numbers $b>a>0$

$$
\begin{aligned}
\int_{\mathbb{S}^{2 N-1}} d \omega \int_{a \leq u \leq b}\left|\varphi_{n}(u, \omega)\right|^{2} d u & \leq \mathrm{e}^{2 N b} \int_{\mathbb{S}^{2 N-1}} d \omega \int_{a \leq u \leq b}\left|\varphi_{n}(u, \omega)\right|^{2} \mathrm{e}^{-2 N u} d u \\
& \leq \mathrm{e}^{2 N b}\left\|\widetilde{u}_{n}\right\|_{L^{2}\left(\mathbb{R}^{2 N}\right)}^{2} \leq \mathrm{e}^{2 N b}\left\|u_{n}\right\|_{L^{2}\left(\mathbb{R}^{2 N}\right)}^{2} \stackrel{n \rightarrow \infty}{\longrightarrow} 0 .
\end{aligned}
$$

Along the same lines, if $\left(h_{n}\right)_{n \in \mathbb{N}}$ is any scale tending to infinity, then we have for any real numbers $b>a>0$ and $n$ large enough

$$
\int_{\mathbb{S}^{2 N-1}} d \omega \int_{a \leq h_{n} u \leq b}\left|\varphi_{n}(u, \omega)\right|^{2} d u \leq \mathrm{e}^{2 N b} \int_{\mathbb{S}^{2 N-1}} d \omega \int_{0 \leq u \leq b}\left|\varphi_{n}(u, \omega)\right|^{2} \mathrm{e}^{-2 N u} d u,
$$

which ensures the result.

Now to extract the log-oscillating components of the sequence $\left(u_{n}\right)$, we shall apply Theorem 2.3 to the sequence $\left(\varphi_{n}\right)$. Up to a subsequence extraction, this gives rise to

$$
\varphi_{n}(t, \omega)=\sum_{j=1}^{\ell}{\widetilde{g_{n}}}^{(j)}(t, \omega)+\widetilde{t}_{n}^{(\ell)}(t, \omega)
$$

for $(t, \omega) \in \mathbb{R}_{+} \times \mathbb{S}^{2 N-1}$, where the sequence $\left({\widetilde{g_{n}}}^{(j)}\right)$ is $h_{n}^{(j)}$-concentrated, with for $j \neq k\left|\log \left(h_{n}^{(j)} / h_{n}^{(k)}\right)\right| \stackrel{n \rightarrow \infty}{\longrightarrow} \infty$, and where $\left(\widetilde{t}_{n}^{(\ell)}\right)$ is unrelated to any scale $\left(h_{n}^{(j)}\right)$ for $j=1, \ldots, \ell$, and satisfies

$$
\limsup _{n \rightarrow \infty} \sup _{j \in \mathbb{Z}} \int_{\mathbb{S}^{2 N-1}} d \omega \int_{2^{j}}^{2^{j+1}}\left|\widetilde{t}_{n}^{(\ell)}(t, \omega)\right|^{2} d t \stackrel{\ell \rightarrow \infty}{\longrightarrow} 0 .
$$

Taking advantage of the above, we deduce that the components ${\widetilde{g_{n}}}^{(j)}$ intervening in Decomposition (3.4) are $h_{n}^{(j)}$-concentrated with $h_{n}^{(j)} \stackrel{n \rightarrow \infty}{\longrightarrow} 0$.

Therefore, up to a subsequence extraction, we have

$$
u_{n}(x)=\sum_{j=1}^{\ell} g_{n}^{(j)}(x)+t_{n}^{(\ell)}(x)+\circ(1),
$$

where the Fourier transforms of the sequences $\left(t_{n}^{(\ell)}\right)$ and $\left(g_{n}^{(j)}\right)$ for $j \in\{1, \ldots, \ell\}$ are supported in $\left\{\xi \in \mathbb{R}^{2 N} ;|\xi| \geq 1\right\}$ and satisfy

$$
\widehat{g_{n}^{(j)}}(\xi)=\frac{1}{|\xi|^{2 N}}{\widetilde{g_{n}}}^{(j)}(\log |\xi|, \omega) \quad \text { and } \quad \widehat{t_{n}^{(\ell)}}(\xi)=\frac{1}{|\xi|^{2 N}} \widetilde{t}_{n}^{(\ell)}(\log |\xi|, \omega),
$$

and where $\circ(1)$ tends to 0 in $H^{N}\left(\mathbb{R}^{2 N}\right)$ and thus in $\mathcal{L}$ as $n$ goes to infinity. Moreover, in light of Proposition A.1 which relates the Orlicz and $\mathcal{B}$ norms

$$
\limsup _{n \rightarrow \infty}\left\|t_{n}^{(\ell)}\right\|_{\mathcal{L}} \stackrel{\ell \rightarrow \infty}{\longrightarrow} 0 \text {. }
$$


It is then obvious that the sequences $\left(|D|^{N} g_{n}^{(j)}\right)$ are $\alpha^{(j)}$ log-oscillating in the sense of Definition 2.1, with $\alpha_{n}^{(j)}=\frac{1}{h_{n}^{(j)}} \stackrel{n \rightarrow \infty}{\longrightarrow} \infty$, and that $\left(|D|^{N} t_{n}^{(\ell)}\right)$ is log-unrelated to the scales $\underline{\alpha}^{(j)}$ for $j=1, \ldots, \ell$. Indeed,

$$
\begin{aligned}
\left.\left.\int_{\frac{1}{\alpha_{n}^{(j)}}(\log |\xi|) \geq R}|| \xi\right|^{N} \widehat{g_{n}^{(j)}}(\xi)\right|^{2} d \xi & =\int_{\mathbb{S}^{2 N-1}} d \omega \int_{\frac{1}{\alpha_{n}^{(j)}}(\log \rho) \geq R}\left|{\widetilde{g_{n}}}^{(j)}(\log \rho, \omega)\right|^{2} \frac{d \rho}{\rho} \\
& =\int_{\mathbb{S}^{2 N-1}} d \omega \int_{\frac{\eta}{\alpha_{n}^{(j)}} \geq R}\left|{\widetilde{g_{n}}}^{(j)}(\eta, \omega)\right|^{2} d \eta .
\end{aligned}
$$

The sequence ${\widetilde{g_{n}}}^{(j)}$ being $\frac{1}{\alpha_{n}^{(j)}}$-concentrated, we deduce that

$$
\left.\left.\limsup _{n \rightarrow \infty} \int_{\frac{1}{\alpha_{n}^{(j)}}(\log |\xi|) \geq R}|| \xi\right|^{N} \widehat{g_{n}^{(j)}}(\xi)\right|^{2} d \xi \stackrel{R \rightarrow \infty}{\longrightarrow} 0 .
$$

Along the same lines, we get

$$
\left.\left.\limsup _{n \rightarrow \infty} \int_{\frac{1}{\alpha_{n}^{(j)}}(\log |\xi|) \leq \frac{1}{R}}|| \xi\right|^{N} \widehat{g_{n}^{(j)}}(\xi)\right|^{2} d \xi \stackrel{R \rightarrow \infty}{\longrightarrow} 0
$$

and establish that $\left(|D|^{N} t_{n}^{(\ell)}\right)$ is log-unrelated to the scales $\underline{\alpha}^{(j)}$ for $j=1, \ldots, \ell$. This achieves the proof of the claim. The fact that $\alpha_{n}^{(j)}=\frac{1}{h_{n}^{(j)}}$ easily implies that the scales $\left(\underline{\alpha}^{(j)}\right)$ are pairwise orthogonal in the sense of (1.5).

3.3. Extraction of cores and profiles. Now our aim is to prove that the sequence $\left(g_{n}^{(j)}\right)_{n \in \mathbb{N}}$ can be decomposed up to a subsequence extraction according to

$$
\sum_{l=1}^{L} \frac{C_{N}}{\sqrt{\alpha_{n}^{(j)}}} \int_{\mathbb{R}^{2 N}} \frac{\mathrm{e}^{i\left(x-x_{n}^{l}\right) \cdot \xi}}{|\xi|^{2 N}} \phi^{l}\left(\frac{\log |\xi|}{\alpha_{n}^{(j)}}\right) d \xi+\circ(1),
$$

where the profiles $\phi^{l}$ belong to $L^{2}\left(\mathbb{R}_{+}\right)$and the cores $\left(x_{n}^{l}\right)_{n \in \mathbb{N}}$ are sequences of points in $\mathbb{R}^{2 N}$ satisfying the orthogonality assumption (1.6), and where $\circ(1)$ designates a sequence which tends to 0 in $\mathcal{L}$ as $n$ and $L$ go to infinity.

For that purpose, let us firstly recall that

$$
g_{n}^{(j)}(x)=\frac{1}{(2 \pi)^{2 N}} \int_{|\xi| \geq 1} \frac{e^{i x \cdot \xi}}{|\xi|^{2 N}}{\widetilde{g_{n}}}^{(j)}(\log |\xi|, \omega) d \xi
$$

where the sequence $\left({\widetilde{g_{n}}}^{(j)}\right)$ is $\frac{1}{\alpha_{n}^{(j)}}$-concentrated. Thus

$$
g_{n}^{(j)}(x)=\frac{C_{N}}{\sqrt{\alpha_{n}^{(j)}}} \int_{|\xi| \geq 1} \frac{\mathrm{e}^{i x \cdot \xi}}{|\xi|^{2 N}} \varphi_{n}^{(j)}\left(\frac{\log |\xi|}{\alpha_{n}^{(j)}}, \omega\right) d \xi
$$

with $\varphi_{n}^{(j)}$ a bounded sequence in $L^{2}\left(\mathbb{R}_{+} \times \mathbb{S}^{2 N-1}\right)$ which is 1-concentrated.

In order to establish (3.8), we shall revisit the proof of Proposition 4.1 of P. Gérard in [13]. For that purpose, with a fixed scale $\left(\alpha_{n}\right)$, let us for a sequence $\left(\varphi_{n}\right)$ bounded 
in the space $L^{2}\left(\mathbb{R}_{+} \times \mathbb{S}^{2 N-1}\right)$ and 1-concentrated denote by $\mathcal{P}\left(\varphi_{n}\right)$ the set of weak limits in $L^{2}\left(\mathbb{R}_{+} \times \mathbb{S}^{2 N-1}\right)$ of subsequences of

$$
\mathrm{e}^{i x_{n} \cdot \mathrm{e}^{\alpha_{n} t} \omega} \varphi_{n}(t, \omega)
$$

where $x_{n} \in \mathbb{R}^{2 N}$ and set

$$
\eta\left(\varphi_{n}\right):=\sup _{\varphi \in \mathcal{P}\left(\varphi_{n}\right)}\|\varphi\|_{L^{2}\left(\mathbb{R}_{+} \times \mathbb{S}^{2 N-1}\right)}^{2}
$$

Now the heart of the matter consists in establishing the following lemma:

Lemma 3.1. Let $\left(\varphi_{n}\right)$ be a bounded sequence in $L^{2}\left(\mathbb{R}_{+} \times \mathbb{S}^{2 N-1}\right)$, 1-concentrated, and $\left(\alpha_{n}\right)_{n \in \mathbb{N}}$ be a sequence of positive real numbers going to infinity. Then, up to a subsequence extraction, there exists a sequence $\left(\Phi^{(l)}\right)_{l \geq 1}$ of functions belonging to $L^{2}\left(\mathbb{R}_{+} \times \mathbb{S}^{2 N-1}\right)$ and a sequence of cores $\left(\underline{x}^{(l)}\right)_{l \geq 1}$, such that for any $L \geq 1$ we have

$$
\varphi_{n}(t, \omega)=\sum_{l=1}^{L} \mathrm{e}^{-i x_{n}^{(l)} \cdot \mathrm{e}^{\alpha_{n} t} \omega} \Phi^{(l)}(t, \omega)+r_{n}^{(L)}(t, \omega)
$$

with

(1) For all $1 \leq l^{\prime}<l$, we have

$-\frac{\log \left|x_{n}^{(l)}-x_{n}^{\left(l^{\prime}\right)}\right|}{\alpha_{n}} \stackrel{n \rightarrow \infty}{\longrightarrow} a \in[-\infty,+\infty[$ with in the case when $a>-\infty$,

$$
\begin{gathered}
\Phi^{(l)}(t, \omega)=0, \quad \text { for } \quad t<a \\
\mathrm{e}^{i x_{n}^{(L)} \cdot \mathrm{e}^{\alpha_{n} t} \omega} r_{n}^{(L)}(t, \omega) \stackrel{n \rightarrow \infty}{\longrightarrow} 0 \quad \text { in } \quad L^{2}\left(\mathbb{R}_{+} \times \mathbb{S}^{2 N-1}\right) .
\end{gathered}
$$

(3) For all $1 \leq l^{\prime}<l$, the sequence

$$
\mathrm{e}^{i\left(x_{n}^{(l)}-x_{n}^{\left(l^{\prime}\right)}\right) \cdot \mathrm{e}^{\alpha_{n} t} \omega} \Phi^{(l)}(t, \omega) \rightarrow 0 \quad \text { in } \quad L^{2}\left(\mathbb{R}_{+} \times \mathbb{S}^{2 N-1}\right), \quad n \rightarrow \infty .
$$

$$
\limsup _{L \rightarrow \infty} \eta\left(r_{n}^{(L)}\right)=0 \text {. }
$$

Remark 3.2. Let us point out that Claims (2) and (3) ensure that for all $l \leq L$, $\mathrm{e}^{i x_{n}^{(l)} \cdot \mathrm{e}^{\alpha_{n} t} \omega} r_{n}^{(L)}(t, \omega) \stackrel{n \rightarrow \infty}{\rightarrow} 0 \quad$ in $L^{2}\left(\mathbb{R}_{+} \times \mathbb{S}^{2 N-1}\right)$ and in particular,

$$
\left\|\theta \varphi_{n}\right\|_{L^{2}}^{2}=\sum_{l=1}^{L}\left\|\theta \Phi^{(l)}\right\|_{L^{2}}^{2}+\left\|\theta r_{n}^{(L)}\right\|_{L^{2}}^{2}+\circ(1), \quad n \rightarrow \infty,
$$

for any $\theta$ in $L^{\infty}\left(\mathbb{R}_{+}\right)$.

Proof. If $\eta\left(\varphi_{n}\right)=0$, we are done. Otherwise there exists $\Phi^{(1)} \in \mathcal{P}\left(\varphi_{n}\right)$ such that

$$
\left\|\Phi^{(1)}\right\|_{L^{2}}^{2} \geq \frac{1}{2} \eta\left(\varphi_{n}\right)
$$

Thus we have, up to a subsequence extraction

$$
\varphi_{n}(t, \omega)=\mathrm{e}^{-i x_{n}^{(1)} \cdot \mathrm{e}^{\alpha_{n} t} \omega} \Phi^{(1)}(t, \omega)+r_{n}^{(1)}(t, \omega),
$$

with $\left(x_{n}^{(1)}\right)$ a core and

$$
\mathrm{e}^{i x_{n}^{(1)} \cdot \mathrm{e}^{\alpha_{n} t} \omega} r_{n}^{(1)}(t, \omega) \stackrel{n \rightarrow \infty}{\rightarrow} 0 \quad \text { in } \quad L^{2}\left(\mathbb{R}_{+} \times \mathbb{S}^{2 N-1}\right)
$$


Now if $\eta\left(r_{n}^{(1)}\right)=0$, we stop the process. If not there exists $\Phi^{(2)} \in \mathcal{P}\left(\varphi_{n}\right)$ such that

$$
\left\|\Phi^{(2)}\right\|_{L^{2}}^{2} \geq \frac{1}{2} \eta\left(r_{n}^{(1)}\right)
$$

which, up to a subsequence extraction, gives rise to

$$
r_{n}^{(1)}(t, \omega)=\mathrm{e}^{-i x_{n}^{(2)} \cdot \mathrm{e}^{\alpha_{n} t} \omega} \Phi^{(2)}(t, \omega)+r_{n}^{(2)}(t, \omega)
$$

for some core $\left(x_{n}^{(2)}\right)$, and with

$$
\mathrm{e}^{i x_{n}^{(2)} \cdot \mathrm{e}^{\alpha_{n} t} \omega} r_{n}^{(2)}(t, \omega) \stackrel{n \rightarrow \infty}{\rightarrow} 0 \quad \text { in } \quad L^{2}\left(\mathbb{R}_{+} \times \mathbb{S}^{2 N-1}\right)
$$

Up to a subsequence extraction, we can suppose that

$$
-\frac{\log \left|x_{n}^{(1)}-x_{n}^{(2)}\right|}{\alpha_{n}} \stackrel{n \rightarrow \infty}{\longrightarrow} a \in[-\infty,+\infty] .
$$

We claim in the case when $a>-\infty$ that $\Phi^{(2)}(t)=0$ for all $t<a$. Indeed, if not there exists $\widetilde{a}<a$ such that

$$
\int_{-\infty}^{\widetilde{a}} \int_{\mathbb{S}^{2 N-1}}\left|\Phi^{(2)}(t, \omega)\right|^{2} d t d \omega=\delta>0 .
$$

By hypothesis if $\epsilon>0$ is chosen so that $\widetilde{a}+\epsilon<a$, then

$$
-\frac{\log \left|x_{n}^{(1)}-x_{n}^{(2)}\right|}{\alpha_{n}} \geq \widetilde{a}+\epsilon, \quad \text { for } n \quad \text { sufficiently large. }
$$

But by construction, we have

$$
\int_{-\infty}^{\tilde{a}} \int_{\mathbb{S}^{2 N-1}}\left|\Phi^{(2)}(t, \omega)\right|^{2} d t d \omega=\lim _{n \rightarrow \infty} \mathcal{I}_{n}
$$

with

$$
\mathcal{I}_{n}:=\int_{-\infty}^{+\infty} \int_{\mathbb{S}^{2 N-1}} \mathrm{e}^{i x_{n}^{(2)} \cdot \mathrm{e}^{\alpha_{n} t} \omega} r_{n}^{(1)}(t, \omega) \chi_{[0, \widetilde{a}]}(t) \overline{\Phi^{(2)}}(t, \omega) d t d \omega
$$

Moreover

$$
\begin{aligned}
\mathcal{I}_{n} & =\int_{-\infty}^{+\infty} \int_{\mathbb{S}^{2 N-1}} \mathrm{e}^{i x_{n}^{(1)} \cdot \mathrm{e}^{\alpha_{n} t} \omega} r_{n}^{(1)}(t, \omega) \mathrm{e}^{i\left(x_{n}^{(2)}-x_{n}^{(1)}\right) \cdot \mathrm{e}^{\alpha_{n} t} \omega} \chi_{[0, \widetilde{a}]}(t) \overline{\Phi^{(2)}}(t, \omega) d t d \omega \\
& =\int_{-\infty}^{+\infty} \int_{\mathbb{S}^{2 N-1}} \mathrm{e}^{i x_{n}^{(1)} \cdot \mathrm{e}^{\alpha_{n} t} \omega} r_{n}^{(1)}(t, \omega) \chi_{[0, \widetilde{a}]}(t) \overline{\Phi^{(2)}}(t, \omega) d t d \omega+\mathcal{R}_{n},
\end{aligned}
$$

with

$$
\begin{aligned}
\left|\mathcal{R}_{n}\right| & \leq \int_{-\infty}^{\widetilde{a}} \int_{\mathbb{S}^{2 N-1}}\left|r_{n}^{(1)}(t, \omega)\right|\left|\Phi^{(2)}(t, \omega)\right|\left|x_{n}^{(2)}-x_{n}^{(1)}\right| \mathrm{e}^{\alpha_{n} t} d t d \omega \\
& \lesssim\left|x_{n}^{(2)}-x_{n}^{(1)}\right| \mathrm{e}^{\alpha_{n} \widetilde{a}} .
\end{aligned}
$$

By virtue of (3.14)

$$
\left|x_{n}^{(1)}-x_{n}^{(2)}\right| \leq \mathrm{e}^{-\alpha_{n}(\widetilde{a}+\epsilon)}, \quad \text { for } n \text { sufficiently large, }
$$

which implies that

$$
\left|\mathcal{R}_{n}\right| \lesssim \mathrm{e}^{-\alpha_{n} \epsilon} \stackrel{n \rightarrow \infty}{\longrightarrow} 0 .
$$


This in light of (3.12) yields a contradiction with (3.13) and then concludes the proof of the orthogonality property for $\Phi^{(2)}$. According to the fact that by construction $\Phi^{(2)}$ is not null, we deduce that $a<+\infty$.

We next address Claim (3), which is in fact a direct consequence of Claim (2). Indeed, in view of the orthogonality property for $\Phi^{(2)}$, it suffices to demonstrate in the case when $a<+\infty$ that for all $g$ in $\mathcal{D}(] a,+\infty\left[\times \mathbb{S}^{2 N-1}\right)$

$$
\mathcal{J}_{n}:=\int_{a}^{+\infty} \int_{\mathbb{S}^{2 N-1}} \mathrm{e}^{i\left(x_{n}^{(1)}-x_{n}^{(2)}\right) \cdot \mathrm{e}^{\alpha_{n} t} \omega} g(t, \omega) d t d \omega \stackrel{n \rightarrow \infty}{\longrightarrow} 0
$$

For that purpose, we perform the change of variables $\xi=\mathrm{e}^{\alpha_{n} t} \cdot \omega$ which leads to

$$
\mathcal{J}_{n}=\frac{1}{\alpha_{n}} \int_{\mathbb{R}^{2 N}} \mathrm{e}^{i\left(x_{n}^{(1)}-x_{n}^{(2)}\right) \cdot \xi} g\left(\frac{\log |\xi|}{\alpha_{n}}, \frac{\xi}{|\xi|}\right) \frac{d \xi}{|\xi|^{2 N}}
$$

where $g\left(\frac{\log |\xi|}{\alpha_{n}}, \frac{\xi}{|\xi|}\right)$ is supported in $\left\{\mathrm{e}^{\alpha_{n} a_{1}} \leq|\xi| \leq \mathrm{e}^{\alpha_{n} b_{1}}\right\}$, with $a<a_{1}<b_{1}<\infty$. Then integrating by parts, we get

$$
\mathcal{J}_{n}=\frac{i}{\alpha_{n}} \int_{\mathbb{R}^{2 N}} \mathrm{e}^{i\left(x_{n}^{(1)}-x_{n}^{(2)}\right) \cdot \xi} \frac{x_{n}^{(1)}-x_{n}^{(2)}}{\left|x_{n}^{(1)}-x_{n}^{(2)}\right|^{2}} \cdot \nabla_{\xi}\left[\frac{1}{|\xi|^{2 N}} g\left(\frac{\log |\xi|}{\alpha_{n}}, \frac{\xi}{|\xi|}\right)\right] d \xi
$$

which implies that

$$
\begin{aligned}
\left|\mathcal{J}_{n}\right| & \lesssim \frac{1}{\alpha_{n}} \int_{\mathrm{e}^{\alpha_{n} a_{1}}}^{\mathrm{e}^{\alpha_{n} b_{1}}} \frac{1}{\left|x_{n}^{(1)}-x_{n}^{(2)}\right|} \frac{\rho^{2 N-1}}{\rho^{2 N+1}} d \rho \\
& \lesssim \frac{\mathrm{e}^{-\alpha_{n} a_{1}}}{\alpha_{n}\left|x_{n}^{(1)}-x_{n}^{(2)}\right|} \stackrel{n \rightarrow \infty}{\longrightarrow} 0,
\end{aligned}
$$

and ensures the result.

An iteration argument allows us to construct the families $\left(\Phi^{(l)}\right)_{l \geq 1}$ and $\left(\underline{x}^{(l)}\right)_{l \geq 1}$ satisfying Claims (1)-(3) of Lemma 3.1. Finally, recalling that by construction

$$
\eta\left(r_{n}^{(L)}\right) \leq 2\left\|\Phi^{(L+1)}\right\|_{L^{2}}^{2},
$$

we deduce in view of the convergence of the series $\sum_{l \in \mathbb{N}}\left\|\Phi^{(l)}\right\|_{L^{2}}^{2}$ that

$$
\limsup _{L \rightarrow \infty} \eta\left(r_{n}^{(L)}\right)=0
$$

This ends the proof of the lemma.

Corollary 3.3. Under notations of Lemma 3.1, we have

$$
\limsup _{n \rightarrow \infty}\left\|R_{n}^{(L)}\right\|_{\mathcal{L}} \stackrel{L \rightarrow \infty}{\longrightarrow} 0
$$

where

$$
R_{n}^{(L)}(x):=\frac{C_{N}}{\sqrt{\alpha_{n}}} \int_{|\xi| \geq 1} \frac{\mathrm{e}^{i x \cdot \xi}}{|\xi|^{2 N}} r_{n}^{(L)}\left(\frac{\log |\xi|}{\alpha_{n}}, \omega\right) d \xi
$$


Proof. The goal is to prove that for all $\varepsilon>0$, there exist $L_{0} \in \mathbb{N}$ and $C$ an absolute constant such that

$$
\forall L \geq L_{0}, \quad \limsup _{n \rightarrow \infty}\left\|R_{n}^{(L)}\right\|_{\mathcal{L}} \leq C \varepsilon .
$$

Recall that $\varphi_{n}$ is a bounded sequence in $L^{2}\left(\mathbb{R}_{+} \times \mathbb{S}^{2 N-1}\right)$ which is 1-concentrated, and therefore there exists $R_{0}>0$ such that

(3.17) $\limsup _{n \rightarrow \infty}\left(\int_{\mathbb{S}^{2 N-1}} \int_{0}^{\frac{1}{R_{0}}}\left|\varphi_{n}(t, \omega)\right|^{2} d t d \omega+\int_{\mathbb{S}^{2 N-1}} \int_{R_{0}}^{\infty}\left|\varphi_{n}(t, \omega)\right|^{2} d t d \omega\right) \leq \varepsilon^{2}$.

Since by Assertion (3.11), we have for any $L \geq 1$

$$
\begin{aligned}
\int_{\mathbb{S}^{2 N-1}} \int_{\left\{t \leq \frac{1}{R_{0}}\right\} \cup\left\{t \geq R_{0}\right\}}\left|r_{n}^{(L)}(t, \omega)\right|^{2} d t d \omega \leq & \int_{\mathbb{S}^{2 N-1}} \int_{\left\{t \leq \frac{1}{R_{0}}\right\} \cup\left\{t \geq R_{0}\right\}}\left|\varphi_{n}(t, \omega)\right|^{2} d t d \omega \\
& +\circ(1),
\end{aligned}
$$

as $n$ tends to infinity, we obtain for any $L \geq 1$

$$
\limsup _{n \rightarrow \infty} \int_{\mathbb{S}^{2 N-1}} \int_{\left\{t \leq \frac{1}{R_{0}}\right\} \cup\left\{t \geq R_{0}\right\}}\left|r_{n}^{(L)}(t, \omega)\right|^{2} d t d \omega \leq \varepsilon^{2} .
$$

Now let us decompose $R_{n}^{(L)}$ into two parts as follows:

$$
R_{n}^{(L)}=\widetilde{R}_{n}^{(L)}+\left(R_{n}^{(L)}-\widetilde{R}_{n}^{(L)}\right),
$$

with

$$
\widetilde{R}_{n}^{(L)}(x):=\frac{C_{N}}{\sqrt{\alpha_{n}}} \int_{|\xi| \geq 1} \frac{\mathrm{e}^{i x \cdot \xi}}{|\xi|^{2 N}} \chi_{R_{0}}\left(\frac{\log |\xi|}{\alpha_{n}}\right) r_{n}^{(L)}\left(\frac{\log |\xi|}{\alpha_{n}}, \omega\right) d \xi
$$

and where $\chi_{R_{0}}$ is a function of $\mathcal{D}(\mathbb{R})$ which is equal to one in $\left\{\frac{1}{R_{0}} \leq y \leq R_{0}\right\}$, supported in $\left\{\frac{1}{2 R_{0}} \leq y \leq 2 R_{0}\right\}$ and valued in $[0,1]$.

First let us consider $R_{n}^{(L)}-\widetilde{R}_{n}^{(L)}$. By straightforward computations, we get

$$
\left\|R_{n}^{(L)}-\widetilde{R}_{n}^{(L)}\right\|_{\mathcal{L}} \lesssim\left\|R_{n}^{(L)}-\widetilde{R}_{n}^{(L)}\right\|_{H^{N}\left(\mathbb{R}^{2 N}\right)} \lesssim\left\|\left(1-\chi_{R_{0}}\right) r_{n}^{(L)}\right\|_{L^{2}}
$$

Since

$$
\left\|\left(1-\chi_{R_{0}}\right) r_{n}^{(L)}\right\|_{L^{2}}^{2} \leq \int_{\left\{t \leq \frac{1}{R_{0}}\right\} \cup\left\{t \geq R_{0}\right\}}\left|r_{n}^{(L)}(t, \omega)\right|^{2} d t d \omega,
$$

we deduce in light of (3.18) that

$$
\limsup _{n \rightarrow \infty}\left\|R_{n}^{(L)}-\widetilde{R}_{n}^{(L)}\right\|_{\mathcal{L}} \lesssim \varepsilon
$$

Now let us address $\widetilde{R}_{n}^{(L)}$. To go to this end, let us start by observing that for any $L \geq 1$

$$
\limsup _{n \rightarrow \infty} \frac{1}{\sqrt{\alpha_{n}}}\left\|\widetilde{R}_{n}^{(L)}\right\|_{L^{\infty}\left(\mathbb{R}^{2 N}\right)} \leq \eta\left(r_{n}^{(L)}\right)^{\frac{1}{2}}\left\|\chi_{R_{0}}\right\|_{L^{2}}
$$

Indeed, if not there exists an integer $L_{0}$, a positive real number $\delta$ and a subsequence $\left(\widetilde{R}_{n_{k}}^{\left(L_{0}\right)}\right)$ such that

$$
\frac{1}{\sqrt{\alpha_{n_{k}}}}\left\|\widetilde{R}_{n_{k}}^{\left(L_{0}\right)}\right\|_{L^{\infty}\left(\mathbb{R}^{2 N}\right)} \geq \eta\left(r_{n_{k}}^{\left(L_{0}\right)}\right)^{\frac{1}{2}}\left\|\chi_{R_{0}}\right\|_{L^{2}}+\delta
$$


for $k$ sufficiently large. Therefore, there exists a sequence of points $\left(x_{n_{k}}\right)$ such that

$$
\frac{1}{\sqrt{\alpha_{n_{k}}}}\left|\widetilde{R}_{n_{k}}^{\left(L_{0}\right)}\left(x_{n_{k}}\right)\right| \geq \eta\left(r_{n_{k}}^{\left(L_{0}\right)}\right)^{\frac{1}{2}}\left\|\chi_{R_{0}}\right\|_{L^{2}}+\frac{\delta}{2}
$$

which means that

$$
\left|\int_{\mathbb{R}_{+}} \int_{\mathbb{S}^{2 N-1}} \chi_{R_{0}}(t) \mathrm{e}^{i x_{n_{k}} \cdot \mathrm{e}^{\alpha_{n_{k}}{ }^{t} \omega}} r_{n_{k}}^{\left(L_{0}\right)}(t, \omega) d t d \omega\right| \geq \eta\left(r_{n_{k}}^{\left(L_{0}\right)}\right)^{\frac{1}{2}}\left\|\chi_{R_{0}}\right\|_{L^{2}}+\frac{\delta}{2},
$$

for $k$ big enough. But the sequence $\left(\mathrm{e}^{i x_{n_{k}} \cdot \mathrm{e}^{\alpha_{n_{k}}{ }^{t} \omega}} r_{n_{k}}^{\left(L_{0}\right)}\right)$ is bounded in $L^{2}\left(\mathbb{R}_{+} \times \mathbb{S}^{2 N-1}\right)$. Thus, up to a subsequence extraction, it converges weakly in $L^{2}$ to a function $H$ belonging to $\mathcal{P}\left(r_{n_{k}}^{\left(L_{0}\right)}\right)$. Passing to the limit, we deduce that

$$
\eta\left(r_{n_{k}}^{\left(L_{0}\right)}\right)^{\frac{1}{2}}\left\|\chi_{R_{0}}\right\|_{L^{2}}+\frac{\delta}{2} \leq\left|\int_{\mathbb{R}_{+}} \int_{\mathbb{S}^{2 N-1}} \chi_{R_{0}}(t) H(t, \omega) d t d \omega\right| \leq\|H\|_{L^{2}}\left\|\chi_{R_{0}}\right\|_{L^{2}}
$$

which contradicts the definition of $\eta\left(r_{n_{k}}^{\left(L_{0}\right)}\right)$ and ends the proof of Claim (3.19). Now using the simple fact that for any function $u$

$$
\int_{\mathbb{R}^{2 N}}\left(\mathrm{e}^{|u(x)|^{2}}-1\right) d x \lesssim \int_{\mathbb{R}^{2 N}} \mathrm{e}^{|u(x)|^{2}}|u(x)|^{2} d x
$$

we get for any $\lambda>0$

$$
\int_{\mathbb{R}^{2 N}}\left(\mathrm{e}^{\left|\frac{\widetilde{R}_{n}^{(L)}(x)}{\lambda}\right|^{2}}-1\right) d x \lesssim \frac{1}{\lambda^{2}} \mathrm{e}^{\frac{\left\|\widetilde{R}_{n}^{(L)}\right\|_{L}^{2}}{\lambda^{2}}}\left\|\widetilde{R}_{n}^{(L)}\right\|_{L^{2}\left(\mathbb{R}^{2 N}\right)}^{2}
$$

But

$$
\left\|\widetilde{R}_{n}^{(L)}\right\|_{L^{2}\left(\mathbb{R}^{2 N}\right)}^{2} \lesssim \int_{\mathbb{S}^{2 N-1}} \int_{\frac{1}{2 R_{0}}}^{2 R_{0}} \mathrm{e}^{-2 N \alpha_{n} t}\left|r_{n}^{(L)}(t, \omega)\right|^{2} d t d \omega \lesssim \mathrm{e}^{-\frac{N \alpha_{n}}{R_{0}}}\left\|r_{n}^{(L)}\right\|_{L^{2}\left(\mathbb{R}_{+} \times \mathbb{S}^{2 N-1}\right)}^{2},
$$

which together with (3.19) and (3.20) implies that

$$
\limsup _{n \rightarrow \infty}\left\|\widetilde{R}_{n}^{(L)}\right\|_{\mathcal{L}} \leq C \eta\left(r_{n}^{(L)}\right)^{\frac{1}{2}}
$$

The fact that $\eta\left(r_{n}^{(L)}\right) \stackrel{L \rightarrow \infty}{\longrightarrow} 0$ allows us to conclude the proof of (3.16).

In order to obtain a decomposition under the form (1.16), we shall replace the profiles obtained above in $L^{2}\left(\mathbb{R}_{+} \times \mathbb{S}^{2 N-1}\right)$ by their average on the sphere. To go to this end, let us point out that if we set

$$
\Phi_{n}^{l}(x):=\frac{C_{N}}{\sqrt{\alpha_{n}}} \int_{\mathbb{R}^{2 N}} \frac{\mathrm{e}^{i\left(x-x_{n}^{l}\right) \cdot \xi}}{|\xi|^{2 N}}\left[\Phi^{(l)}\left(\frac{\log |\xi|}{\alpha_{n}}, \omega\right)-\phi^{l}\left(\frac{\log |\xi|}{\alpha_{n}}\right)\right] d \xi
$$

where $\phi^{l}(t):=\frac{1}{\omega_{2 N-1}} \int_{\mathbb{S}^{2 N-1}} \Phi^{(l)}(t, \omega) d \omega$, then we have

$$
\left\|\Phi_{n}^{l}\right\|_{\mathcal{L}} \stackrel{n \rightarrow \infty}{\longrightarrow} 0 .
$$

Assertion (3.21) stems from the following lemma: 
Lemma 3.4. Let $\left(\alpha_{n}\right)_{n \in \mathbb{N}}$ be a sequence of positive real numbers going to infinity, $\Phi$ be a function in $L^{2}\left(\mathbb{R}_{+} \times \mathbb{S}^{2 N-1}\right)$ and set for $x \in \mathbb{R}^{2 N}$

$$
\Phi_{n}(x):=\frac{1}{(2 \pi)^{2 N}} \sqrt{\frac{1}{\alpha_{n}}} \int_{\mathbb{R}^{2 N}} \frac{\mathrm{e}^{i x \cdot \xi}}{|\xi|^{2 N}}\left[\Phi\left(\frac{\log |\xi|}{\alpha_{n}}, \omega\right)-\phi\left(\frac{\log |\xi|}{\alpha_{n}}\right)\right] d \xi
$$

where

$$
\phi(t):=\frac{1}{\omega_{2 N-1}} \int_{\mathbb{S}^{2 N-1}} \Phi(t, \omega) d \omega
$$

Then

$$
\left\|\Phi_{n}\right\|_{\mathcal{L}} \stackrel{n \rightarrow \infty}{\longrightarrow} 0
$$

Proof. Let us first consider the case when $\Phi$ belongs to $\mathcal{D}\left(\mathbb{R}_{+} \times \mathbb{S}^{2 N-1}\right)$ and decompose $\Phi_{n}(x)$ as follows:

$$
\begin{gathered}
\Phi_{n}(x)=\Phi_{n}^{(1)}(x)+\Phi_{n}^{(2)}(x), \quad \text { with } \\
\Phi_{n}^{(1)}(x):=\frac{1}{(2 \pi)^{2 N}} \sqrt{\frac{1}{\alpha_{n}}} \int_{1 \leq|\xi| \leq \max \left(\frac{1}{|x|}, 1\right)} \frac{\mathrm{e}^{i x \cdot \xi}}{|\xi|^{2 N}}\left[\Phi\left(\frac{\log |\xi|}{\alpha_{n}}, \omega\right)-\phi\left(\frac{\log |\xi|}{\alpha_{n}}\right)\right] d \xi .
\end{gathered}
$$

Observe that in view of $(3.22)$ we have

$$
\sqrt{\frac{1}{\alpha_{n}}} \int_{1 \leq|\xi| \leq \max \left(\frac{1}{|x|}, 1\right)} \frac{1}{|\xi|^{2 N}}\left[\Phi\left(\frac{\log |\xi|}{\alpha_{n}}, \omega\right)-\phi\left(\frac{\log |\xi|}{\alpha_{n}}\right)\right] d \xi=0 .
$$

Therefore

$$
\begin{aligned}
\left|\Phi_{n}^{(1)}(x)\right|= & \frac{1}{(2 \pi)^{2 N}} \sqrt{\frac{1}{\alpha_{n}}} \mid \int_{1 \leq|\xi| \leq \max \left(\frac{1}{|x|}, 1\right)} \frac{\left(\mathrm{e}^{i x \cdot \xi}-1\right)}{|\xi|^{2 N}}\left[\Phi\left(\frac{\log |\xi|}{\alpha_{n}}, \omega\right)\right. \\
& \left.-\phi\left(\frac{\log |\xi|}{\alpha_{n}}\right)\right] d \xi \mid \\
\lesssim & \frac{|x|}{\sqrt{\alpha_{n}}} \int_{1}^{\max \left(\frac{1}{|x|}, 1\right)} \int_{\mathbb{S}^{2 N-1}}\left|\Phi\left(\frac{\log \rho}{\alpha_{n}}, \omega\right)-\phi\left(\frac{\log \rho}{\alpha_{n}}\right)\right| d \rho d \omega \\
\lesssim & \frac{1}{\sqrt{\alpha_{n}}} .
\end{aligned}
$$

Now to estimate $\Phi_{n}^{(2)}$, we shall argue as for the proof of Assertion (3.15). Thus integrating by parts, we deduce that $\Phi_{n}^{(2)}(x)=\Phi_{n}^{(2,1)}(x)+\Phi_{n}^{(2,2)}(x)$, with

$$
\begin{aligned}
\Phi_{n}^{(2,1)}(x)= & \frac{i}{(2 \pi)^{2 N}} \sqrt{\frac{1}{\alpha_{n}}} \int_{|\xi| \geq \max \left(\frac{1}{|x|}, 1\right)} \mathrm{e}^{i x \cdot \xi} \frac{x}{|x|^{2}} \cdot \nabla_{\xi}\left[\frac { 1 } { | \xi | ^ { 2 N } } \left(\Phi\left(\frac{\log |\xi|}{\alpha_{n}}, \omega\right)\right.\right. \\
& \left.\left.-\phi\left(\frac{\log |\xi|}{\alpha_{n}}\right)\right)\right] d \xi, \\
\left\|\Phi_{n}^{(2,2)}\right\|_{L^{\infty}} \lesssim & \frac{1}{\sqrt{\alpha_{n}}} .
\end{aligned}
$$


By straightforward computations, one can prove that

$$
\left|\Phi_{n}^{(2,1)}(x)\right| \lesssim \frac{1}{\sqrt{\alpha_{n}}|x|} \int_{\rho \geq \max \left(\frac{1}{|x|}, 1\right)} \frac{d \rho}{\rho^{2}} \lesssim \frac{1}{\sqrt{\alpha_{n}}} .
$$

In summary, in the case when $\Phi$ belongs to $\mathcal{D}\left(\mathbb{R}_{+} \times \mathbb{S}^{2 N-1}\right)$, we have proved that

$$
\left\|\Phi_{n}\right\|_{L^{\infty}} \lesssim \frac{1}{\sqrt{\alpha_{n}}}
$$

This achieves the proof of the result, noticing that $\left\|\Phi_{n}\right\|_{L^{2}} \stackrel{n \rightarrow \infty}{\longrightarrow} 0$ and remembering that for bounded functions the Orlicz space $\mathcal{L}$ acts like $L^{2}$.

Let us now treat the case when $\Phi \in L^{2}\left(\mathbb{R}_{+} \times \mathbb{S}^{2 N-1}\right)$. Density arguments ensure that for any $\varepsilon>0$, there exists $\Phi_{\varepsilon} \in \mathcal{D}\left(\mathbb{R}_{+} \times \mathbb{S}^{2 N-1}\right)$ such that

$$
\left\|\Phi-\Phi_{\varepsilon}\right\|_{L^{2}} \leq \varepsilon \text {. }
$$

Therefore $\varepsilon>0$ being fixed, we can write

$$
\Phi_{n}(x)=\frac{1}{(2 \pi)^{2 N}} \sqrt{\frac{1}{\alpha_{n}}} \int_{\mathbb{R}^{2 N}} \frac{\mathrm{e}^{i x \cdot \xi}}{|\xi|^{2 N}}\left[\Phi_{\varepsilon}\left(\frac{\log |\xi|}{\alpha_{n}}, \omega\right)-\phi_{\varepsilon}\left(\frac{\log |\xi|}{\alpha_{n}}\right)\right] d \xi+t_{n, \varepsilon}(x),
$$

where $\phi_{\varepsilon}(t):=\frac{1}{\omega_{2 N-1}} \int_{\mathbb{S}^{2 N-1}} \Phi_{\varepsilon}(t, \omega) d \omega$ and $\left\|t_{n, \varepsilon}\right\|_{H^{N}\left(\mathbb{R}^{2 N}\right)} \lesssim \varepsilon$. This ends the proof of the result by virtue of the Sobolev embedding (1.3).

Remark 3.5. Let us also note that

$$
\left\|\Phi_{n}\right\|_{\dot{H}^{N}\left(\mathbb{R}^{2 N}\right)}^{2}=\|\Phi\|_{L^{2}\left(\mathbb{R}_{+} \times \mathbb{S}^{2 N-1}\right)}^{2}-\omega_{2 N-1}\|\phi\|_{L^{2}\left(\mathbb{R}_{+}\right)}^{2} .
$$

Now let us return to the proof of Theorem 1.6. In view of the above analysis, up to a subsequence extraction, we obtain a decomposition of $\left(u_{n}\right)$ under the form (3.5), and for any $j \geq 1$ we have

$$
g_{n}^{(j)}(x)=\sum_{k=1}^{K} \frac{C_{N}}{\sqrt{\alpha_{n}^{(j)}}} \int_{\mathbb{R}^{2 N}} \frac{\mathrm{e}^{i\left(x-x_{n}^{(j, k)}\right) \cdot \xi}}{|\xi|^{2 N}} \phi^{(j, k)}\left(\frac{\log |\xi|}{\alpha_{n}^{(j)}}\right) d \xi+\mathcal{R}_{n}^{(j, k)}(x),
$$

where the couples $\left(\underline{x}^{(j, k)}, \phi^{(j, k)}\right)$ are pairwise orthogonal in the sense of (1.6), and where

$$
\begin{gathered}
\limsup _{n \rightarrow \infty}\left\|\mathcal{R}_{n}^{(j, K)}\right\|_{\mathcal{L}} \stackrel{K \rightarrow \infty}{\longrightarrow} 0, \quad \text { and } \\
\left\|g_{n}^{(j)}\right\|_{\dot{H}^{N}\left(\mathbb{R}^{2 N}\right)}^{2}=\sum_{k=1}^{K}\left\|\phi^{(j, k)}\right\|_{L^{2}\left(\mathbb{R}_{+}\right)}^{2}+\left\|\mathcal{R}_{n}^{(j, k)}\right\|_{\dot{H}^{N}\left(\mathbb{R}^{2 N}\right)}^{2}+\circ(1), \quad n \rightarrow \infty .
\end{gathered}
$$

Summing Decomposition (3.24), we deduce that up to a subsequence extraction

$$
u_{n}(x)=\sum_{j=1}^{L} \sum_{k=1}^{K_{j}} \frac{C_{N}}{\sqrt{\alpha_{n}^{(j)}}} \int_{\mathbb{R}^{2 N}} \frac{\mathrm{e}^{i\left(x-x_{n}^{(j, k)}\right) \cdot \xi}}{|\xi|^{2 N}} \phi^{(j, k)}\left(\frac{\log |\xi|}{\alpha_{n}^{(j)}}\right) d \xi+r_{n}^{\left(L, k_{1}, \ldots, K_{L}\right)}(x),
$$


with under notation of (3.5) and (3.24)

$$
\mathrm{r}_{n}^{\left(L, k_{1}, \ldots, K_{L}\right)}=t_{n}^{(L)}+\sum_{j=1}^{L} \mathcal{R}_{n}^{\left(j, K_{j}\right)}
$$

Since $\left(|D|^{N} t_{n}^{(L)}\right)$ is log-unrelated to any scale $\left(\alpha_{n}^{(j)}\right), j=1, \ldots, L$, and $\left(|D|^{N} g_{n}^{(j)}\right)$ is $\alpha^{(j)} \log$-oscillating where the scales $\alpha^{(j)}$ are pairwise orthogonal in the sense of (1.5), we deduce from (2.5) and (3.26) that for any $L$ and $K_{j}$

$$
\left\|u_{n}\right\|_{\dot{H}^{N}\left(\mathbb{R}^{2 N}\right)}^{2}=\sum_{j=1}^{L} \sum_{k=1}^{K_{j}}\left\|\phi^{(j, k)}\right\|_{L^{2}\left(\mathbb{R}_{+}\right)}^{2}+\left\|r_{n}^{\left(L, k_{1}, \ldots, K_{L}\right)}\right\|_{\dot{H}^{N}\left(\mathbb{R}^{2 N}\right)}^{2}+\circ(1), \quad n \rightarrow \infty,
$$

which concludes the proof of the theorem.

\section{Appendix A}

A.1. Connection between $\mathcal{B}$ and Orlicz spaces. The following result allows us to relate the Orlicz norm with the $\|\cdot\|_{\mathcal{B}}$ norm:

Proposition A.1. There is a positive constant $C$ such that

$$
\|w\|_{\mathcal{L}} \leq C\|\widetilde{w}\|_{\mathcal{B}}
$$

where

$$
w(x):=\frac{1}{(2 \pi)^{2 N}} \int_{|\xi| \geq 1} \frac{\mathrm{e}^{i x \cdot \xi}}{|\xi|^{2 N}} \widetilde{w}(\log |\xi|, \omega) d \xi,
$$

with $\widetilde{w}$ in $L^{2}\left(\mathbb{R}_{+} \times \mathbb{S}^{2 N-1}\right)$ and

$$
\|\widetilde{w}\|_{\mathcal{B}}:=\sup _{j \in \mathbb{Z}}\left(\int_{\mathbb{S}^{2 N-1}} d \omega \int_{2^{j}}^{2^{j+1}}|\widetilde{w}(t, \omega)|^{2} d t\right)^{\frac{1}{2}}
$$

Proof. For fixed $\lambda>0$, let us estimate the integral:

$$
\int_{\mathbb{R}^{2 N}}\left(\mathrm{e}^{\left|\frac{w(x)}{\lambda}\right|^{2}}-1\right) d x
$$

Obviously

$$
\int_{\mathbb{R}^{2 N}}\left(\mathrm{e}^{\left|\frac{w(x)}{\lambda}\right|^{2}}-1\right) d x=\sum_{p \geq 1} \frac{\|w\|_{L^{2 p}}^{2 p}}{\lambda^{2 p} p !} .
$$

Firstly let us investigate $\|w\|_{L^{2 p}}^{2 p}$. We have that for any $p \geq 1$

$$
\|w\|_{L^{2 p}}^{2 p} \leq C^{2 p}\|\widehat{w}\|_{L^{\frac{2 p}{2 p-1}}}^{2 p} .
$$

But in view of (A.1) and Hölder inequality, we get for any $p \geq 2$

$$
\|\widehat{w}\|_{L^{\frac{2 p}{2 p-1}}}^{\frac{2 p}{2 p-1}} \lesssim\|\widetilde{w}\|_{\mathcal{B}}^{\frac{2 p}{2 p-1}} \sum_{j \in \mathbb{Z}}\left(\int_{2^{j}}^{2^{j+1}} \mathrm{e}^{-\frac{2 N t}{p-1}} d t\right)^{\frac{p-1}{2 p-1}}
$$


Clearly,

$$
\left(\int_{2^{j}}^{2^{j+1}} \mathrm{e}^{-\frac{2 N t}{p-1}} d t\right)^{\frac{p-1}{2 p-1}}=\left(\frac{p-1}{2 N}\right)^{\frac{p-1}{2 p-1}}\left(\mathrm{e}^{\frac{-2 N 2^{j}}{p-1}}-\mathrm{e}^{\frac{-2 N 2^{j+1}}{p-1}}\right)^{\frac{p-1}{2 p-1}}
$$

Choosing $j_{0}$ such that $\frac{1}{2} \leq \frac{2^{j_{0}}}{2 p-1} \leq 1$, we infer that

$$
\sum_{j \geq j_{0}}\left(\int_{2^{j}}^{2^{j+1}} \mathrm{e}^{-\frac{2 N t}{p-1}} d t\right)^{\frac{p-1}{2 p-1}} \lesssim(2 p-1)^{\frac{p-1}{2 p-1}}
$$

Indeed observing that the ratio $\frac{p-1}{2 p-1}$ is uniformly bounded with respect to $p \geq 2$, we deduce that for all $j$

$$
\left(\int_{2^{j}}^{2^{j+1}} \mathrm{e}^{-\frac{2 N t}{p-1}} d t\right)^{\frac{p-1}{2 p-1}} \lesssim(2 p-1)^{\frac{p-1}{2 p-1}} \mathrm{e}^{-\frac{2 N 2^{j}}{2 p-1}}
$$

which according to the choice of $j_{0}$ gives rise to

$$
\begin{aligned}
\sum_{j \geq j_{0}}\left(\int_{2^{j}}^{2^{j+1}} \mathrm{e}^{-\frac{2 N t}{p-1}} d t\right)^{\frac{p-1}{2 p-1}} & \lesssim(2 p-1)^{\frac{p-1}{2 p-1}} \sum_{j \geq j_{0}} \mathrm{e}^{-\frac{2 N 2^{j}}{2 p-1}} \\
& \lesssim(2 p-1)^{\frac{p-1}{2 p-1}} \sum_{j \geq j_{0}}(2 p-1) 2^{-j} \\
& \lesssim(2 p-1)^{\frac{p-1}{2 p-1}}(2 p-1) 2^{-j_{0}} \lesssim(2 p-1)^{\frac{p-1}{2 p-1}}
\end{aligned}
$$

On the other hand for $j \leq j_{0}$, we get

$$
\begin{aligned}
\left(\int_{2^{j}}^{2^{j+1}} \mathrm{e}^{-\frac{2 N t}{p-1}} d t\right)^{\frac{p-1}{2 p-1}} & =\left(\frac{p-1}{2 N}\right)^{\frac{p-1}{2 p-1}} \mathrm{e}^{-\frac{2 N 2^{j}+1}{2 p-1}}\left(\mathrm{e}^{\frac{2 N 2^{j}}{p-1}}-1\right)^{\frac{p-1}{2 p-1}} \\
& \lesssim(2 p-1)^{\frac{p-1}{2 p-1}}\left(\frac{2^{j}}{2 p-1}\right)^{\frac{p-1}{2 p-1}} \lesssim\left(2^{j}\right)^{\frac{p-1}{2 p-1}}
\end{aligned}
$$

In view of the choice of $j_{0}$, this implies that

$$
\sum_{j \leq j_{0}}\left(\int_{2^{j}}^{2^{j+1}} \mathrm{e}^{-\frac{2 N t}{p-1}} d t\right)^{\frac{p-1}{2 p-1}} \lesssim \sum_{j \leq j_{0}}\left(2^{\frac{p-1}{2 p-1}}\right)^{j} \lesssim 2^{\left(j_{0}+1\right) \frac{p-1}{2 p-1}} \lesssim(2 p-1)^{\frac{p-1}{2 p-1}}
$$

By virtue of the above, this ensures that

$$
\sum_{j \in \mathbb{Z}}\left(\int_{2^{j}}^{2^{j+1}} \mathrm{e}^{-\frac{2 N t}{p-1}} d t\right)^{\frac{p-1}{2 p-1}} \lesssim(2 p-1)^{\frac{p-1}{2 p-1}}
$$

We deduce that for any $p \geq 2$

$$
\|w\|_{L^{2 p}}^{2 p} \lesssim C^{2 p}\|\widetilde{w}\|_{\mathcal{B}}^{2 p}(2 p-1)^{p-1} .
$$


Along the same lines, we obtain

$$
\|w\|_{L^{2}}^{2} \lesssim\|\widetilde{w}\|_{\mathcal{B}}^{2}
$$

which leads to

$$
\int_{\mathbb{R}^{2 N}}\left(\mathrm{e}^{\left|\frac{w(x)}{\lambda}\right|^{2}}-1\right) d x \lesssim \sum_{p \geq 1} \frac{C^{2 p}\|\widetilde{w}\|_{\mathcal{B}}^{2 p}(2 p-1)^{p-1}}{\lambda^{2 p} p !} .
$$

This ends the proof of the proposition thanks to Stirling formula.

A.2. Fourier approximation of generalizations of example by Moser. The aim of this section is to establish Proposition 1.7 which allows us to relate the generalizations of example by Moser to the elementary concentrations involving in Decomposition (1.16).

Proof of Proposition 1.7. The proof of Proposition 1.7 goes along the same lines as the proof of Lemma 3.4. We sketch it here for the convenience of the reader. Before entering into the details, let us point out that the function

$$
\widetilde{g}_{n}(x):=\widetilde{C}_{N} \sqrt{\alpha_{n}} \psi\left(\frac{-\log |x|}{\alpha_{n}}\right)
$$

is supported in the unit ball of $\mathbb{R}^{2 N}$ and also writes:

$$
\tilde{g}_{n}(x)=\frac{C_{N}}{\sqrt{\alpha_{n}}} \int_{1 \leq|\xi| \leq \frac{1}{|x|}} \frac{1}{|\xi|^{2 N}} \varphi\left(\frac{\log |\xi|}{\alpha_{n}}\right) d \xi
$$

Now by density arguments, we can as in the proof of Lemma 3.4 reduce to the case when $\varphi$ belongs to $\mathcal{D}\left(\mathbb{R}_{+}\right)$. Let us then decompose $g_{n}(x)$ as follows:

$$
\begin{gathered}
g_{n}(x)=g_{n}^{(1)}(x)+g_{n}^{(2)}(x), \quad \text { with } \\
g_{n}^{(1)}(x):=\frac{C_{N}}{\sqrt{\alpha_{n}}} \int_{1 \leq|\xi| \leq \max \left(\frac{1}{|x|}, 1\right)} \frac{\mathrm{e}^{i x \cdot \xi}}{|\xi|^{2 N}} \varphi\left(\frac{\log |\xi|}{\alpha_{n}}\right) d \xi .
\end{gathered}
$$

In one hand in view of (A.2), we have

$$
\begin{gathered}
g_{n}^{(1)}(x)=\widetilde{g}_{n}(x)+t_{n}^{1}(x), \quad \text { with } \\
\left|t_{n}^{1}(x)\right|=\frac{C_{N}}{\sqrt{\alpha_{n}}}\left|\int_{1 \leq|\xi| \leq \max \left(\frac{1}{|x|}, 1\right)} \frac{\left(\mathrm{e}^{i x \cdot \xi}-1\right)}{|\xi|^{2 N}} \varphi\left(\frac{\log |\xi|}{\alpha_{n}}\right) d \xi\right| \\
\lesssim \frac{|x|}{\sqrt{\alpha_{n}}} \int_{1}^{\max \left(\frac{1}{|x|}, 1\right)}\left|\varphi\left(\frac{\log \rho}{\alpha_{n}}\right)\right| d \rho \lesssim \frac{1}{\sqrt{\alpha_{n}}} .
\end{gathered}
$$

On the other hand integrating by parts, we get $g_{n}^{(2)}(x)=g_{n}^{(2,1)}(x)+g_{n}^{(2,2)}(x)$ with

$$
\begin{aligned}
g_{n}^{(2,1)}(x) & =\frac{i C_{N}}{\sqrt{\alpha_{n}}} \int_{|\xi| \geq \max \left(\frac{1}{|x|}, 1\right)} \mathrm{e}^{i x \cdot \xi} \frac{x}{|x|^{2}} \cdot \nabla_{\xi}\left[\frac{1}{|\xi|^{2 N}} \varphi\left(\frac{\log |\xi|}{\alpha_{n}}\right)\right] d \xi \quad \text { and } \\
\left\|g_{n}^{(2,2)}\right\|_{L^{\infty}} & \lesssim \frac{1}{\sqrt{\alpha_{n}}} .
\end{aligned}
$$


Clearly

$$
\left|g_{n}^{(2,1)}(x)\right| \lesssim \frac{1}{\sqrt{\alpha_{n}}|x|} \int_{\rho \geq \max \left(\frac{1}{|x|}, 1\right)} \frac{d \rho}{\rho^{2}} \lesssim \frac{1}{\sqrt{\alpha_{n}}} .
$$

This easily ensures the result.

\section{References}

[1] S. Adachi and K. Tanaka, Trudinger type inequalities in $\mathbb{R}^{N}$ and their best exponents, Proc. Amer. Math. Soc. 128(7) (2000), 2051-2057.

[2] D.R. Adams, A sharp inequality of J. Moser for higher order derivatives, Ann. Math., 128(2) (1988), 385-398.

[3] H. Bahouri and P. Gérard, High frequency approximation of solutions to critical nonlinear wave equations, Amer. J. Math, 121 (1999), 131-175.

[4] H. Bahouri, M. Majdoub and N. Masmoudi, On the lack of compactness in the 2D critical Sobolev embedding, J. Funct. Anal., 260 (2011), 208-252.

[5] H. Bahouri, A. Cohen and G. Koch, A general wavelet-based profile decomposition in the critical embedding of function spaces, Confluentes Math., 3 (3) (2011), 1-25.

[6] H. Bahouri, M. Majdoub and N. Masmoudi, Lack of compactness in the 2D critical Sobolev embedding, the general case, Notes aux Comptes-Rendus de l'Académie des Sciences de Paris, Ser. I 350, (2012), 177-181.

[7] H. Bahouri, M. Majdoub and N. Masmoudi, Lack of compactness in the 2D critical Sobolev embedding, the general case, J. Math. Pures Appl., 101 (2014), 415-457.

[8] H. Bahouri, On the elements involving in the lack of compactness in critical Sobolev embedding, Concentration Analysis and Applications to PDE, Trends in Mathematics, (2013), 1-15.

[9] H. Bahouri and I. Gallagher, On the stability in weak topology of the set of global solutions to the Navier-Stokes equations, Arch. Ration. Mech. Anal., 209(2) (2013), 569-629.

[10] I. Ben Ayed and M. K. Zghal, Characterization of the lack of compactness of $H_{\text {rad }}^{2}\left(\mathbb{R}^{4}\right)$ into the Orlicz space, to appear in Commun. Contemp. Math.

[11] H. Brézis and J.-M. Coron, Convergence of solutions of H-systems or how to blow bubbles, Arch. Ration. Mech. Anal., 89 (1985), 21-86.

[12] I. Gallagher, G. Koch and F. Planchon, A profile decomposition approach to the $L_{t}^{\infty}\left(L_{x}^{3}\right)$ NavierStokes regularity criterion, Math. Annal., 355 (2013), 1527-1559.

[13] P. Gérard, Description du défaut de compacité de l'injection de Sobolev, ESAIM Contrôle Optimal et Calcul des Variations, 3 (1998), 213-233.

[14] S. Jaffard, Analysis of the lack of compactness in the critical Sobolev embeddings, J. Funct. Anal., 161 (1999), 384-396.

[15] C.E. Kenig and F. Merle, Global well-posedness, scattering and blow-up for the energy critical focusing non-linear wave equation, Acta Math., 201 (2008), 147-212.

[16] S. Keraani, On the defect of compactness for the Strichartz estimates of the Schrödinger equation, J. Differ. Equ., 175 (2) (2001), 353-392.

[17] S. Kesavan, Symmetrization \& applications, Series in Analysis, 3, World Scientific Publishing, Hackensack, NJ, 2006.

[18] C. Laurent, On stabilization and control for the critical Klein-Gordon equation on a 3-D compact manifold, J. Funct. Anal., 260 (5) (2011), 1304-1368.

[19] E.H. Lieb and M. Loss, Analysis, in 'Graduate Studies in Mathematics', American Mathematical Society, 14, (1997), xviii+278 pp.

[20] P.-L. Lions, The concentration-compactness principle in the calculus of variations. The limit case. I., Rev. Mat. Iberoamericana 1(1) (1985), 145-201.

[21] P.-L. Lions, The concentration-compactness principle in the calculus of variations. The limit case. II., Rev. Mat. Iberoamericana, 1(2) (1985), 45-121.

[22] J. Moser, A sharp form of an inequality of N. Trudinger, Indiana University Math. J. 20 (1971), 1077-1092.

[23] B. Ruf, A sharp Trudinger-Moser type inequality for unbounded domains in $\mathbb{R}^{2}$, J. Funct. Anal., 219 (2005), 340-367. 
[24] B. Ruf and F. Sani, Sharp Adams-type inequalities in $\mathbb{R}^{n}$, J. Amer. Math. Soc. 365 (2013), 645-670.

[25] M. Struwe, Critical points of embeddings of $H_{0}^{1, n}$ into Orlicz spaces, Annales de l'Institut Henri Poincaré, Analyse Non Linéaire, 5 (1988), 425-464.

[26] T. Tao, Global regularity for a logarithmically supercritical defocusing nonlinear wave equation for spherically symmetric data, J. Hyperbolic Differ. Equ., 4 (2007), 259-265.

Laboratoire D'Analyse et de Mathématiques Appliquées UMR 8050, Université ParisEst Créteil, 61, avenue du Général de Gaulle, 94010 Créteil Cedex, France

E-mail address: hbahouri@math.cnrs.fr

E-mail address: galina.perelman@u-pec.fr 